\title{
BOREL STRUCTURES AND INVARIANTS FOR MEASURABLE TRANSFORMATIONS
}

\section{JACOB FELDMAN ${ }^{1}$}

ABSTRACT. It is shown that, in a certain sense, there is no possibility of getting a complete set of conjugacy-invariants for measure-preserving transformations of a probability space.

1. Introduction. One of the central problems of erogodic theory has been to find a complete set of conjugacy-invariants for the Lebesgue measure-preserving transformations of the unit interval or for some reasonably large subclass thereof. A recent important breakthrough by Ornstein [8] is the theorem that the Kolmogorov-Sinai entropy is a complete invariant for the Bernoulli transformations. On the other hand, in the larger class of Kolmogorov transformations, Ornstein and Shields [10] constructed an uncountable family $\mathcal{K}$ of mutually nonconjugate transformations all having the same entropy. In the present paper, it is shown that in a certain sense there is no possibility of getting a complete set of conjugacy-invariants for the measurepreserving transformations, or even for the family $\mathcal{K}$. Qualifications like "a.e." will be frequently and shamelessly omitted in the subsequent exposition.

2. Topologies on measurable transformations. I now describe several natural topologies on measure-preserving transformations. This will all be done on the unit interval $C$ with Lebesgue measure $\lambda$. Since it is only the measure-theoretic structure of $(C, \lambda)$ which concerns us, it would be inappriate to topologize the transformations by any of the standard topologies on transformations considered as real-valued functions.

Consider first the operators of norm $\leq 1$ on $L_{2}(\lambda)$. These form a Polish (= separable and completely metrizable) space in the strong operator topology.

Received by the editors October 25, 1973.

AMS (MOS) subject classifications (1970). Primary 28A65; Secondary 47A35, $54 \mathrm{H} 26$.

Key words and phrases. Ergodic theory, $K$-automorphism, Borel structure, invariants.

1 This work was supported in part by NSF Grant GP-15735. 
The weak operator topology is strictly coarser; however, it is easily verified that the Borel sets are the same for both topologies. Multiplication is continuous for the strong operator topology on isometries, or indeed on any uniformly bounded set of operators.

The isometries form a closed subset in the strong operator topology. The unitaries, i.e. the invertible isometries, form a Borel subset in the weak topology, and inversion is weakly continuous, since $U$ is unitary if and only if $U^{*} U=I$, in which case $U^{*}=U^{-1}$. Furthermore, the weak and strong topologies agree on the unitaries: see [4] for this and some of the foregoing facts.

Next, consider partial isometries of the form $U_{T} f=f \circ T$ for a measurepreserving transformation $T$ of the unit interval. Since they may be characterized by the property $U(f g)=(U f)\left(U_{g}\right)$ for bounded $f$, they form a strongly closed and, therefore, Borel subset of the isometries. Thus the previous topologies may be transferred to the set of measure-preserving transformations, and the Borel structure induced is standard in the sense of Mackey [7]. When I speak of the Borel structure on measure-preserving transformations, this is the Borel structure which is meant. The set of all invertible measure-preserving transformations on $C$ will be called $T$, and the Borel sets will be called $\mathfrak{T}$.

In [5], Halmos makes $\mathbf{T}$ into a topological group in two different ways. One way just induces the aforementioned topology; however, as a topological group, $\mathbf{T}$ is complete. This is what Halmos calls the weak topology on $\mathbf{T}$. A pleasant consequence is the following:

2.1 Proposition. The conjugacy class of each $T \in \mathbf{T}$ is a Borel set.

Proof. The map $(S, T) \mapsto S T S^{-1}$ is jointly continuous, since inversion is continuous in $\mathbf{T}$ and multiplication is jointly continuous. Then, since $\mathbf{T}$ is a Polish space, each conjugacy class is a Borel set [2].

The second topology used in [5] is there called the uniform topology; this is implemented by the invariant distance function $d(S, T)=$ $\sup _{A} \lambda(S A \triangle T A)$. This makes $\mathbf{T}$ into a nonseparable complete metric group. The uniform topology is finer than the previous one, and consequently the Borel sets for this topology (which I will call uniform Borel sets, and denote by $\mathfrak{U}$ ) include the previous Borel sets, and in fact the inclusion is strict. However, we have the following:

2.2 Proposition. For each $a \geq 0$, the set $\mathbf{N}_{a}(T)=\{S: d(S, T) \leq a\} \in \mathfrak{I}$. 
Proof. Choose a dense sequence of measurable sets $A_{1}, A_{2}, \cdots \subset C$. Then it is easy to see that $\mathbf{N}_{a}(T)=\left\{S: \lambda\left(S A_{n} \Delta T A_{n}\right) \leq a\right.$ for all $\left.n\right\}=$ $\bigcap_{n}\left\{S: \lambda\left(S A_{n} \triangle T A_{n}\right) \leq a\right\}$, which is a closed set for the weak topology.

2.3 Remark. It is not difficult to see that the $\sigma$-algebra generated by the $\mathbf{N}_{a}(T)$ includes the uniform Baire sets (= the $\sigma$-algebra generated by the uniform compact sets); and we have just seen that it is a subalgebra of $\mathfrak{T}$. Questions. Is it a proper subalgebra? Are the uniform Baire sets a proper subalgebra?

Finally, the uniform topology on $T$ should not be confused with the topology induced by the uniform operator topology. The uniform operator topology merely induces the discrete topology on $\mathbf{T}$.

3. Transformations as measures on shift space. Let $\mathscr{P}_{\infty}$ be the set of all countable measurable ordered partitions $P=\left(P^{1}, P^{2}, \ldots\right)$ of $C$. Also let $\mathcal{P}_{k}$ be the set of partitions with $k$ elements. Then we have injections $\mathcal{P}_{1}^{k} \rightarrow \mathscr{P}_{2} \rightarrow \cdots \rightarrow \mathscr{P}_{k} \rightarrow \cdots \rightarrow \mathcal{P}_{\infty}$, by sending $\left(P^{1}, P^{2}, \cdots, P^{k}\right)$ in $\mathcal{P}_{k}$ to $\left(P^{1}, \ldots, P_{k}, \varnothing\right)$ in $\mathcal{P}_{k+1}$ or to $\left(P^{1}, \ldots, P^{k}, \varnothing, \ldots\right)$ in $P_{\infty}$. Here $\varnothing$ is the empty set. The set $\mathscr{P}_{\infty}$ is given the metric $d(P, Q)=1 / 2 \Sigma_{i} \lambda\left(P^{i} \Delta Q^{i}\right)=$ $\lambda\left(\cup_{i} P^{i} \Delta Q^{i}\right)$. Each $\mathscr{P}_{k}$ is a closed subset of $\mathscr{P}_{\infty}$, and $\mathscr{P}^{\prime} \bigcup_{k<\infty} \mathscr{P}_{k}$ is a dense $F_{\sigma} \cdot \mathscr{P}_{\infty}$ is a Polish space. Finally: $\mathscr{P}_{\infty}$ can be regarded as a subset of $\mathbb{Q}^{N}$, where $\mathbb{C}$ is the measure algebra of $(C, \lambda)$ and $N$ is the positive integers. The product topology thereby induced on $\mathcal{P}_{\infty}$ is identical with its original topology. The Borel sets of $\mathscr{P}_{\infty}$ will be called $\mathfrak{P}_{\text {. }}$.

Now let $M$ be the one point compactification of $N$. Let $\Lambda$ be the set of all two-sided infinite sequences with values in $M$. With the product topology, $\Lambda$ is a compact metric space. The subset $\Omega=\{\omega: \omega(n)<\infty$ for all $n\}$ is a $G_{\delta}$ in $\Lambda$. The shift is continuous on $\Lambda$, and sends $\Omega$ to itself. Another way of viewing invertible measure-preserving transformations is as Borel probability measures on $\Lambda$ which give measure 1 to $\Omega$ and are invariant under the shift.

Let $\Lambda_{k}$ be all sequences $\left(n_{-k}, n_{-k+1}, \cdots, n_{k}\right)$ of elements of $M$. Let $\rho_{k}$ be the restriction map from $\Lambda$ to $\Lambda_{k}$, and let $\sigma_{k}$ be the restriction map from $\Lambda_{k+1}$ to $\Lambda_{k}$. Then for any Borel probability measure $\mu$ on $\Lambda$ we have a measure $\mu_{k}=\mu \circ \rho_{k}^{-1}$ on $\Lambda_{k}$ for each $k$, and $\mu_{k}=\mu_{k+1} \circ \sigma_{k}^{-1}$. Conversely, to each sequence of probability measures $\mu_{k}$ on $\Lambda_{k}$ such that $\mu_{k}=$ $\mu_{k+1} \circ \sigma_{k+1}^{-1}$, there exists a unique $\mu$ on $\Lambda$ such that $\mu \circ \rho_{k}^{-1}=\mu_{k}$ for all $k$. (This is just the Kolmogorov extension theorem in a simple case.) Further, $\mu$ will give measure 1 to $\Omega$ if and only if each $\mu_{k}$ gives measure 1 to each $\Omega_{k}$. 
Let $\mathbb{M}(\Lambda)$ be the Borel probability measures on $\Lambda$. Since $\Omega$ is a Borel subset of $\Lambda$, we may regard $\pi(\Omega)$ as a subset of $\pi(\Lambda)$. First consider a topology on $\pi(\Lambda)$ regarded as a subset of the dual of $\mathcal{C}(\Lambda)$ : the weak* topology. This is a Polish topology (see, for example, [12]). $\pi(\Omega)$ is a Borel set, in fact a $G_{\delta}$, since it is

$$
\bigcap_{j=-\infty}^{\infty} \bigcap_{k=1}^{\infty} \bigcap_{l=1}^{\infty}\left\{\mu \in M(\Lambda): \int 1[-l, l]{ }^{(\omega(j))} d \mu(\omega)>1-\frac{1}{k}\right\}
$$

and $\omega \mapsto 1[-l, l](\omega(j))$ is continuous for each $j, l$. The weak* Borel sets of $\mathbb{M}(\Omega)$ will be called $\mathbb{M}$.

The weak* topology on probability measures is also called the "vague" topology; and for a function on probability measures to be Borel measurable for this topology may be interpreted, in the present context, as its being computable as a limit of the values of the measure on sets of $\Omega$ involving only finitely many coordinates.

Now a map $\Phi$ will be introduced from $T \times \mathcal{P}_{\infty}$ to $\pi(\Omega)$, as follows: $\Phi(T, P)$ is the unique Borel probability measure $\mu$ on $\Omega$ súch that $\mu \circ P_{k}^{-1}\left\{\left(n_{-k}, \cdots, n_{k}\right)\right\}=\lambda\left(\bigcap_{j=-k}^{k} T^{j} P^{n_{j}}\right) . \Phi(T, P)$ will sometimes be called $\mu_{T, P}$. The $T$-invariance of $\lambda$ implies shift-invariance of $\mu_{T, P}$.

3.1 Proposition. $\Phi: T \times \mathcal{P}_{\infty} \rightarrow \mathbb{M}(\Omega)$ is continuous, for the weak ${ }^{*}$ topology on $\pi(\Omega)$ and the weak topology on $\mathrm{T}$.

Proof. First it will be shown that for each $\left(n_{-k}, \cdots, n_{k}\right)$ in $\Omega_{k}$ the numerical function $(T, P) \mapsto \lambda\left(\bigcap_{j=-k}^{k} T^{j} P^{n} j\right)$ is continuous. Let $(S, Q)$ be another pair in $\mathbf{T} \times \mathcal{P}_{\infty}$. Then, setting $A_{i}=\bigcap_{j=i}^{k} T^{j} P^{n}$, and $B_{i}=$ $\bigcap_{j=-k}^{i} S^{j} Q^{n_{j}}$, we have

$$
\left|\lambda\left(\bigcap_{j=-k}^{k} T^{j} P^{n} j\right)-\lambda\left(\bigcap_{j=-k}^{k} S^{j} Q^{n_{j}}\right)\right|
$$

$$
\begin{aligned}
& \left|\lambda\left(A_{-k}\right)-\lambda\left(B_{k}\right)\right| \leq \sum_{i=-k}^{k-1}\left|\lambda\left(B_{i-1} \cap A_{i}\right)-\lambda\left(B_{i} \cap A_{i+1}\right)\right| \\
\leq & \sum_{i=-k}^{k-1} \lambda\left(\left(B_{i-1} \cap A_{i}\right) \Delta\left(B_{i} \cap A_{i+1}\right)\right)
\end{aligned}
$$

since $|\lambda(C)-\lambda(D)| \leq \lambda(C \triangle D)$ in general. Now set $C_{i}=B_{i-1} \cap A_{i+1}$. Then $B_{i-1} \cap A_{i}=C_{i} \cap T^{i} P^{n_{i}}$, while $B_{i} \cap A_{i+1}=C_{i} \cap S^{i} Q^{n_{i}}$. Thus the last term in the sequence of estimates is 


$$
\sum_{i=-k}^{k-1} \lambda\left(\left(C_{i} \cap T^{i} P^{n}\right) \Delta\left(C_{i} \cap S^{i} Q^{n}{ }^{n}\right)\right) \leq \sum_{i=-k}^{k-1} \lambda\left(T^{i} P^{n} i \Delta S^{i} Q^{n}{ }^{n}\right),
$$

since $\lambda((C \cap A) \Delta(C \cap B)) \leq \lambda(A \triangle B)$ in general. Now:

$$
\lambda\left(T^{i} P^{{ }^{n}}{ } \Delta S^{i} P^{n}{ }^{i}\right)+\lambda\left(S^{i} P^{n}{ }^{i} \Delta S^{i} Q^{n}{ }^{i}\right)=\lambda\left(T^{i} P^{n}{ }^{i} \Delta S^{i} P^{n}{ }^{i}\right)+\lambda\left(P^{n}{ }^{i} \Delta Q^{n}{ }^{i}\right) .
$$

Fix $P$. The first term will be small if $T$ is weakly close to $S$, while the second will be small if $Q$ is close to $P$ in $\mathcal{P}_{\infty}$.

Now: let $f$ be any continuous real-valued function on $\Lambda_{k}$, and bounded by 1 . Then:

$$
\int_{\Lambda} f \circ \rho_{k} d \mu_{T, P}=\int_{\Lambda_{k}} f d \mu_{T, P} \circ \rho_{k}^{-1}=\int_{\Omega_{k}} f d \mu_{T, P} \circ \rho_{k}^{-1}
$$

Fix $l$ so large that, setting $\Xi=\left\{\left(-n_{k}, \cdots, n_{k}\right)\right.$ : all $\left.n_{j} \leq l\right\}$, one has $\mu_{T, P} \circ \rho_{k}^{-1}(\Xi)>1-\epsilon$. Choose $(S, Q)$ sufficiently close to $(T, P)$ that $\mu_{S, Q} \circ \rho_{k}^{-1}$ differs from $\mu_{T, P} \circ \rho_{k}^{-1}$ on each of the $l^{2 k+1}$ elements of $\Xi$ by less than $\epsilon / l^{2 k+1}$. Then also $\mu_{S, Q} \circ \rho_{k}^{-1}(\Xi)>1-\epsilon$. Also, let $g=1_{\Xi} f$. Then $g$ is still continuous, and

$$
\begin{aligned}
&\left|\int f d \mu_{T, P} \circ \rho_{k}^{-1}-\int f d \mu_{S, Q} \circ \rho_{k}^{-1}\right| \\
& \leq\left|\int g d \mu_{T, P} \circ \rho_{k}^{-1}-\int g d \mu_{S, Q} \circ \rho_{k}^{-1}\right| \\
&+\mu_{T, P} \circ \rho_{k}^{-1}\left(\Lambda_{k} / \Xi\right)+\mu_{S, Q} \circ \rho_{k}^{-1}\left(\Lambda_{k} / \Xi\right) \\
& \leq \sum_{n \in \Xi}|g(n)|\left|\mu_{P, T} \circ \rho_{k}^{-1}(n)-\mu_{S, Q} \circ \rho_{k}^{-1}(n)\right|+2 \epsilon \leq 3 \epsilon .
\end{aligned}
$$

A finer topology is given by considering "weak convergence of measures" in $\pi(\Omega)$. This is the topology defined by requiring continuity of the functions $\mu \mapsto \int_{\Omega} f d \mu$ for all bounded and continuous functions on $\Omega$. Since such functions do not necessarily extend to continuous functions on $\Lambda$, this topology could be finer; in fact, it is, and makes $M(\Omega)$ into a nonseparable completely metrizable space. The Borel structure for this topology is thus very fine; call it $\Re$. However:

3.2 Proposition. The $\sigma$-algebra generated by the functionals $\mu \mapsto \int_{\Omega} f d \mu, f$ bounded and continuous on $\Omega$, is precisely $M$.

Proof. First, let $f$ be bounded and continuous on $\Omega$. Let $f_{n}(\omega)=f\left(\omega_{n}\right)$, where $\omega_{n}(j)$ is $\omega(j)$ if $j \leq n$, and $\omega(j)=1$ if $j>n$. Then $\omega_{n} \rightarrow \omega$ in $\Omega$, 
so $f_{n}(\omega) \rightarrow f(\omega)$, for each $\omega \in \Omega$. Now:

$$
\int_{\mathbf{\Omega}} f d \mu-\int_{\mathbf{\Omega}} f d \mu_{0}=\lim _{n \rightarrow \infty} \int_{\mathbf{\Omega}} f_{n} d \mu-\int_{\mathbf{\Omega}} f_{n} d \mu_{0} .
$$

Thus, $\left|\int_{\mathbf{\Omega}} f d \mu-\int_{\mathbf{\Omega}} f d \mu_{0}\right| \leq a \Leftrightarrow$ for each $l>0,3 m$ such that $\mid \int_{\mathbf{\Omega}} f_{n} d \mu-$ $\int_{\Omega} f_{n} d \mu_{0} \mid \leq a+1 / l$ for all $m>n$. So,

$\left\{\mu=\left|\int_{\Omega} f d \mu-\int_{\Omega} f d \mu_{0}\right|<a\right\}=\bigcap_{l=1}^{\infty} \bigcup_{m=1}^{\infty} \bigcap_{n=m}^{\infty}\left\{\mu:\left|\int_{\Omega} f d \mu-\int_{\Omega} f d \mu_{0}\right| \leq a+\frac{1}{l}\right\}$,

which is a weak* $F_{\sigma \delta}$, and therefore in $\mathbb{M}$.

Conversely: consider only those functions on $\Omega$ which extend continuously to the compact set $\Lambda$. The functions $\mu \mapsto \int_{\Lambda} f d \mu$ separate $)(\Lambda)$, so polynomials in them are uniformly dense in all weak ${ }^{*}$ continuous functions on $\pi(\Lambda)$ (by Stone-Weierstrass). Thus, the $\sigma$-algebra generated by such functions $\mu \mapsto \int_{\Lambda} f d \mu$ is all of the weak* Borel sets in $\mathbb{M}(\Lambda)$, since $\mathbb{M}(\Lambda)$ is a Polish space. Consequently, looking only at $\mu \in \mathbb{M}(\Omega)$, we see that $\int_{\mathbf{\Lambda}} f d \mu=\int_{\mathbf{\Omega}} f d \mu$ and the functions $\mu \mapsto \int_{\mathbf{\Omega}} f d \mu$ generate all of $\Re$.

3.3 Remark. The distinction between the two topologies arises only because we are permitting an infinite "alphabet". Let $\pi_{n}(\Omega)$ be those measures which are supported on the closed subset $\{\omega: \omega(j) \leq n$ for all $j\}$ of $\Lambda$. Then the weak and weak* topologies have the same restriction to $\pi_{n}(\Omega)$, which is itself a weak* closed subset of $\pi(\Omega)$. See [12] for details.

4. Borel structures of various subsets. Define the entropy $h$ on $\mathfrak{P}_{\infty}$ by $h(P)=-\sum_{i=1}^{k} \lambda\left(P_{i}\right) \log \lambda\left(P_{i}\right)$; then $h$ is a nonnegative, upper semicontinuous function on $\mathcal{P}_{\infty}$, finite on $\mathcal{P}$ and continuous on each $\mathcal{P}_{k}$.

The map $T \mapsto T^{-1} P$ is a continuous map from the weak topology on transformations to $\mathcal{P}_{\infty}$. Finally, the map: $\mathcal{P}_{\infty} \times \mathcal{P}_{\infty} \rightarrow \mathcal{P}_{\infty}$ given by sending $(P, Q)$ to $P \vee Q$ is jointly continuous. Thus the map $T \mapsto$ $h\left(\bigvee_{j}^{m=1} T^{-j} P\right)$ is $T$-measurable.

Then so is $\lim _{n \rightarrow \infty}(1 / n) b\left(\bigvee_{j=0}^{n-1} T^{-j} P\right)=H(P, T)$, the entropy of $P$ with respect to $T$. Now choose a fixed increasing sequence $P_{k}$ of finite partitions such that $\bigcup_{k=1}^{\infty} P_{k}$ generates the measurable sets of $C$. Then the Kolmogorov-Sinai theorem [1] says the entropy of $T$, call it $H(T)$, may be obtained as $\lim _{k \rightarrow \infty} H\left(P_{k}, T\right)$. Thus:

\subsection{Proposition. Entropy is $\mathfrak{T}$-measurable on T.}

Now consider the set of ergodic transformations. These may be described in terms of the corresponding unitary operators: $T$ is erogodic if and only if the only eigenfunctions of $U_{T}$ whose eigenvalue is 1 are the con- 
stant functions. Alternately, in terms of the mean ergodic theorem, $T$ is ergodic if and only if $\lim _{n \rightarrow \infty}(1 / n)\left(1+\cdots+U_{T}^{n}\right)=E$, where $E$ is the onedimensional projection on the space of constant functions, and the limit is in the strong topology. But $U \mapsto(1 / n)\left(I+\cdots+U^{n}\right)$ is a strongly continuous function from isometries to isometries, so the limit is a Borel function, and consequently:

4.2 Proposition. The set of ergodic transformations is in $\mathfrak{T}$.

4.3 Proposition. The set of Kolmogorov transformations is in $\mathfrak{T}$.

Proof. Recall that $\mathfrak{T}$ is standard. Then one can use the indirect method of showing that the Kolmogorov transformations form both an analytic set and a coanalytic set, so that by [6] they form a set in $\mathfrak{T}$.

Consider the following condition on pairs $(T, P)$ :

(a) For each integer $m>0$ there is an integer $N$ such that for all $n \geq$ $N$ and all $k>0, \bigvee_{i=m+1}^{n+m} T^{-i} P$ is $(1 / m)$-independent of $\bigvee_{i=1}^{k} T^{i} P$.

Recall the definition of $\epsilon$-independence: $Q$ is said to be $\epsilon$-independent of $R$ if the total measure of those sets $R_{j}$ for which $\lambda\left(R_{j}\right) \neq 0$ and $\Sigma_{i}\left|\lambda\left(Q_{i} \cap R_{j}\right) / \lambda\left(R_{j}\right)-\lambda\left(Q_{i}\right)\right| \geq \epsilon$ is less than $\epsilon$. Thus, $\{(Q, R): Q$ is $\epsilon$-independent of $R\}$ is a Borel subset of $\mathscr{P}_{\infty} \times \mathscr{P}_{\infty}$, and since both the maps $T \mapsto$ $\bigvee_{i=m+1}^{n+m} T^{-i} P$ and $T \mapsto \bigvee_{i=1}^{k} T^{i} P$ are Borel maps, it follows that the pairs $(T, P)$ in $\mathbf{T} \times \mathcal{P}_{\infty}$ which satisfy (a) form a set in $\mathfrak{T} \times \mathfrak{P}$.

Now: one characterization of the class $A$ of Kolmogorov transformations is as $\{T: \forall P \in \mathcal{P},(T, P)$ satisfies (a) $\}$. Thus, $\mathbf{A}$ is an analytic set.

Another characterization is as $\{T: \exists$ generator $P \in \mathcal{P}$ such that $(T, P)$ satisfies (a) $\}$. Thus, if it can be shown that $\{(T, P): P$ is a generator for $T\}$ is in $\mathfrak{T} \times \mathfrak{P}$, it will follow that $A$ is a coanalytic set, and the proof will be complete. This is done in the next proposition.

4.4 Proposition. $\left\{(T, P): T \in \mathrm{T}, P \in \mathcal{P}_{\infty}, P\right.$ is a generator for $\left.T\right\}$ is $a G_{\delta}$ (for the weak topology on $\mathrm{T}$ ).

Proof. Choose a fixed dense sequence $A_{1}, A_{2}, \ldots$ of measurable sets of $C$. Then $P$ is a generator for $T$ if and only if for each $j$ and $k$ there exists $l$ and a set of sequences $\left(n_{-l}, \cdots, n_{l}\right)$ with each $n_{i} \leq l$, such that the union over this set of sequences of the sets $\bigcap_{j=-l}^{l} T^{j} P^{n_{j}}$ has symmetric difference with $A_{k}$ of measure $<1 / j$.

4.5 Proposition. The set $\mathbf{B}$ of Bernoulli transformations of finite entropy is in $\mathfrak{T}$.

Sketch of proof. An analogous line of reasoning can be used: since $T$ 
is Bernoulli with finite entropy if and only if there exists an independent finite generator, B forms a coanalytic set. On the other hand, $T$ is Bernoulli if and only if every finite generator is very weakly Bernoulli in the sense of [9]. (This is a result of Ornstein and Weiss [11].) This description makes it possible to exhibit $\mathbf{B}$ as an analytic set, too.

5. Remarks about invariants. One view is that a reasonable invariant for a set $X$ of objects must be realizable as a real-valued Borel function for some naturally arising Borel structure on $X$. This, of course, cannot be a theorem; it is simply a requirement which gains plausibility from examples.

Another sort of invariant, however, is obtained where one uses one complicated mathematical object to classify another. For instance: conjugacy of $T_{1}$ and $T_{2}$ implies unitary equivalence of $U_{T_{1}}$ and $U_{T_{2}}$. Thus, unitary invariants for unitary operators may be used as conjugacy invariants for invertible measurable transformations. Unfortunately, the invariants under unitary equivalence are given in terms of equivalence classes of measures under mutual absolute continuity. The results of [3] seem to imply that these cannot be converted into nice Borel functions.

At any rate, and with whatever justification, I will insist that the invariants be $\mathfrak{U}$-measurable on $\mathbf{T}$; note that this is weaker than saying that they are $\mathfrak{T}$-measurable on $T$.

By a complete set of conjugacy invariants will be meant a countable family of conjugacy invariants which, between them, can distinguish between any pair of nonconjugate transformations. (Obviously, one wants countability; else, why not use the indicator functions of the conjugacy classes?)

One more notion. Consider the example of matrices under similarity. The Jordan canonical form provides a complete set of invariants in terms of some complex numbers and some integers. These can be given in such a way that for all possible values of the invariants there is a corresponding class of matrices. It is, however, conceivable that a set of mathematical objects could have a complete set of invariants which are nice Borel functions, but nevertheless, these invariants could not be given in such a way that all possible values of the invariants corresponded to an actual class of objects. If the stronger property holds, let us call the set of invariants free; otherwise, restricted. For example, if $T_{1}$ and $T_{2}$ are conjugate, then $U_{T_{1}}$ and $U_{T_{2}}$ are unitarily equivalent, so that the unitary invariants for unitary operators may be used as a set (not complete, of course) of invariants for the invertible measure-preserving transformations. Thus, the spectrum $\sigma\left(U_{T}\right)$ is a conjugacy-invariant for $T$. This is possibly a quite 
reasonable sort of invariant, but it is far from known what closed subsets of the unit circle can arise as $\sigma\left(U_{T}\right)$ for some $T$.

The reader will immediately see that while the existence of a free set of invariants for $X$ need not imply existence of such a set for a subset $Y$ $C X$, existence of a complete set of invariants for $X$ gives a complete set for $Y$.

5.1 Theorem. There does not exist even a restricted complete set of conjugacy-invariants for the measure-preserving transformations of the unit interval with Lebesgue measure, or even for the Kolmogorov transformations of a given fixed finite entropy.

The proof will be given in the next section.

6. The Omstein-Shields construction. Here is a very brief description of those aspects of the construction in [10] which will be relevant here. For each function $g$ from the positive integers to $\{0,1\}$, a transformation $T_{\boldsymbol{g}}$ is constructed, with each $T_{\boldsymbol{g}} \in \mathbf{A}$, and $T_{\boldsymbol{g}}$ will be conjugate to $T_{g^{\prime}}$ if and only if $g(n)=g^{\prime}(n)$ for all but finitely many integers $n$. All the $T_{g}^{g}$ have the same entropy. The map $g \mapsto T_{g}$ will be a Borel map from the obvious Borel structure on the functions $g$ to measurable transformations. The transformations in [10] were not placed on the unit interval, and the purpose of these remarks is to put them there.

There were also constructed in [10] partitions $P_{g}$, and actually the aforementioned results apply rather to the restriction of $T_{g}$ to the invariant $\sigma$-algebra generated by $P_{g}$ under $T_{g}$. However, as is remarked there, a very minor change in the construction can be made which will have the effect of making each $P_{g}$ a generator of the full $\sigma$-algebra of Lebesgue measurable sets; and it is such a version of the construction which will be utilized here.

Let $\Gamma_{n}$ be all functions from $\{1, \ldots, n\}$ to $\{0,1\}$, and $\Gamma_{\infty}$ all functions from the positive integers to $\{0,1\}$. For each $g \in \Gamma_{\infty}$, let $\gamma_{n}(g)=$ $g\{1, \cdots, n\} \in \Gamma_{n}$. For each $\gamma \in \Gamma_{n}$, denote by $\gamma \epsilon$, where $\epsilon=0$ or 1 , the element of $\Gamma_{n+1}$ which agrees with $\gamma$ on $\{1, \ldots, n\}$ and equals $\epsilon$ on $n$ +1 . For each $n$, and each $\gamma \in \Gamma_{n}$, there will be an interval $C_{\gamma}=\left[0, c_{\gamma}\right]$ on the real line, which is divided into $h(\gamma)$ subintervals $I_{1}^{\gamma}, \ldots, I_{h(\gamma)}^{\gamma}$ of equal length; they are not necessarily labeled in ascending order. $D_{\gamma}$ will denote $I_{1}^{\gamma} \cup \cdots \cup I_{h(\gamma)-1}^{\gamma}$. A gadget (called $G(n)$ in [10], but actually depending on $\gamma$ ) is defined by

(1) the transformation $S_{\gamma}: D_{\gamma} \rightarrow C_{\gamma}$ which sends $I_{i}^{\gamma}$ onto $I_{i+1}^{\gamma}$ by 
translation, $i=1, \cdots, h(\gamma)-1$, and which, for convenience, we extend to all of $C_{\gamma}$, for example by sending $I_{h(\gamma)}^{\gamma}$ by translation onto $I_{1}^{\gamma}$;

(2) a certain three element partition $Q_{\gamma}$ of $D_{\gamma}$, which for convenience we extend to all of $C_{\gamma}$, for example, by putting all of $I_{h(\gamma)}^{\gamma}$ into $Q_{\gamma}^{1}$.

The following properties hold, for any $\gamma$ and $\epsilon=0$ or 1 :

(a) $D_{\gamma \epsilon} \supset D_{\gamma}$,

(b) $S_{\gamma \epsilon}\left|D_{\gamma}=S_{\gamma}\right| D_{\gamma}$,

(c) $Q_{\gamma \epsilon}\left|D_{\gamma}=Q_{\gamma}\right| D_{\gamma}$.

Also, for any $g$,

(d) $h\left(\gamma_{n}(g)\right) \uparrow \infty$,

(e) $c\left(\gamma_{n}(g)\right)$ is bounded above.

Since the Lebesgue measure $\lambda\left(D_{\gamma_{n(g)}}\right) \uparrow$, by (a), and since $c\left(\gamma_{n}(g)\right)=$ $\lambda\left(D_{\gamma_{n}(g)}\right)+1 / h\left(\gamma_{n}(g)\right)$, it follows that $c\left(\gamma_{n}(g)\right)$ converges to some $c(g)$, while $\lambda\left(D_{\gamma_{n}(g)}\right) \uparrow c(g)$. Notice that $[0, c(g))=\bigcup_{n} C_{\gamma_{n}(g)}=\bigcup_{n} D_{\gamma_{n}(g)}$.

The whole apparatus is now transferred to $[0,1]$ by scaling down: let $S_{\gamma}$ be the map $x \mapsto c(\gamma)_{x}$, and $S_{g}$ the map $S \mapsto c(g)_{x}$. Then define $T_{\gamma}$ : $C \rightarrow C$ by $T_{\gamma}=s_{\gamma}^{-1} S_{\gamma} s_{\gamma}$, and $Q_{\gamma}$ by $P_{\gamma}$, where $P_{\gamma}^{i}=s_{\gamma}^{-1}\left(Q_{\gamma}^{i}\right), i=1$, 2, 3. Since $C\left(\gamma_{n}(g)\right) \rightarrow C(g)$ for all $g \in \Gamma_{\infty}$, it easily follows that $T_{\gamma_{n}(g)}$ converges in the uniform topology to a transformation $T_{g}$, which may alternately be described as $s_{\boldsymbol{g}}^{-1} S_{\boldsymbol{g}} s_{\boldsymbol{g}}$, where $S_{\boldsymbol{g}}:[0, c(g)) \rightarrow[0, c(g))$ is defined by $S_{g}\left|D_{\gamma_{n}(g)}=S_{\gamma_{n}(g)}\right| D_{\gamma_{n}(g)}$, a definition which is consistent, by (b). Similarly, $\quad P_{\gamma_{n}(g)}$ converges in $\mathcal{P}_{3}$ to a 3-element partition of $[0,1]$, which may alternately be described by $P_{g}^{i}=s_{g}^{-1}\left(Q_{g}^{i}\right)$, where $Q_{g}^{i}=\bigcup_{n} Q_{\gamma_{n}(g)}^{i} \cap$ $D_{\gamma_{n}}$.

Each $g \mapsto T_{\gamma_{n}(g)}$ is a $\mathfrak{l}$-measurable function: $\Gamma_{\infty} \rightarrow \mathrm{T}$, so the limit $g \mapsto T_{g}$ is likewise. Similarly, $g \mapsto P_{g}$ is a $\Re_{P}$-measurable function: $\Gamma_{\infty} \rightarrow$ $\mathfrak{P}_{3}$.

6.1 Proposition. The set $\mathbf{K}=\left\{T_{g}: g \in \Gamma_{\infty}\right\}$ is in $\mathfrak{T}$.

Proof. $K$ is a countable-to-one image of the standard Borel space $\Gamma_{\infty}$ in the standard Borel space $T$. The mapping function, $g \mapsto T_{g}$, is $\mathfrak{U}$-measurable, hence $\mathfrak{T}$-measurable. So, by [6], the image is Borel.

Now for the proof of Theorem 5.1 of the previous section. In fact, here is a somewhat stronger result.

6.2 Theorem. Let $\mathbf{S}$ be any $\mathfrak{H}$-measurable subset of $\mathbf{K}$ which contains a complete set of representatives from $\mathbf{K}$. Let $\phi_{1}, \phi_{2}, \cdots$ be a sequence $\phi$ of real-valued $\mathfrak{U}$-measurable functions on $\mathbf{K}$ each of which is constant on 
equivalence classes. Then there exists an uncountable subset of inequivalent elements of $\mathbf{S}$ on which each of the $\phi_{j}$ is constant.

Proof. Without loss of generality, the $\phi_{j}$ may be combined into a single real-valued $\mathfrak{U}$-measurable function $\phi$.

Let $\Sigma=\left\{g: T_{g} \in \mathrm{S}\right\}$. Let $g_{0}, g_{1}, \ldots$ be a listing of the members of $\Gamma_{\infty}$ which are 0 from some point on, with $g_{0} \equiv 0$. Let $\Sigma_{n}=\Sigma+g_{n}$, so $\Sigma_{0}=\Sigma$. Let $\psi_{n}\left(g+g_{n}\right)=\phi\left(T_{g}\right)$. If $g+g_{n}=h+g_{m}$, then $T_{g} \sim T_{b}$, so $\phi\left(T_{g}\right)=\phi\left(T_{h}\right)$; thus $\psi_{n}=\psi_{m}$ on $\Sigma_{n} \cap \Sigma_{m}$. Now, since $g \mapsto T_{g}$ is a (uniformly) Borel map, it follows that each $\Sigma_{n}$ is a Borel set and each $\psi_{n}$ is a Borel function. Furthermore, $\bigcup_{n} \Sigma_{n}=\Gamma_{\infty}$, by our assumptions on $\mathrm{S}$, so the function $\psi$ defined by setting $\psi=\psi_{n}$ on $\Sigma_{n}$ is a Borel function, and is constant on equivalence classes in $\Gamma_{\infty}$.

It will be shown that $\psi$ must give uncountable many equivalence classes the same value. As a consequence, $\psi_{0}$ must take the same value on uncountably many inequivalent $g$, so $\psi$ must do the same on the corresponding $T_{\boldsymbol{g}}$.

The argument that $\psi$ must give the same value to uncountably many equivalence classes is essentially due to Blackwell, and goes as follows: Let $p$ be coin-tossing measure on $\Gamma_{\infty}$. Then the restriction of $p$ to the equivalence-saturated Borel subsets of $\Gamma_{\infty}$, the "tail $\sigma$-field", takes on only the values of 0 and 1 , by the Borel-Cantelli lemma. Since $\psi$ is both Borel measurable and constant on equivalence classes, $p \circ \psi^{-1}$ is a $0-1$ valued Borel measure on the reals. Thus, its support is a single point $x_{0}$. Therefore, the countable set $\psi^{-1}\left(x_{0}\right)$ has $p$-measure one, which is impossible because $p$ is a continuous measure.

Here is a corresponding theorem on shift space. Let $\mu_{g}=\Phi\left(T_{g}, P_{g}\right)$. Let $\mathcal{K}=\left(\mu_{\boldsymbol{g}}: g \in \Gamma_{\infty}\right\}$. Then $g \mapsto \mu_{\boldsymbol{g}}$ is a Borel map from the standard space $\Gamma_{\infty}$ into the standard space $M(\Omega)$ with the weak* topology. Since it is at most countable-to-one, $\mathcal{K}$ is in $M$. Incidentally: each $\mu \in \mathcal{K}$ has its support on $\{\omega: \omega(j) \leq 3$ for all $j\}$; but the weak* and weak topologies agree on the set of all such probability measures, so that on $\mathcal{K}$ the weak and weak* topologies (and therefore Borel structures) agree, and thus the restrictions of $M$ and $\Re$ to this set of measures agree.

6.3 Theorem. Let $\mathcal{S} \subset \mathcal{K}$ and $\in \Re$. Assume that $\mathcal{S}$ contains a complete set of representatives of $\mathcal{K}$. Let $\theta_{1}, \theta_{2}, \cdots$ be any sequence of real-valued $\Re$-measurable functions on $\mathcal{S}$ which are constant on equivalence classes. Then there is an uncountable set of mutually inequivalent elements of $\mathcal{S}$, on which each $\theta_{j}$ is constant. 
Proof. Essentially the same as before: let $\Sigma=\left\{g: \mu_{g} \in \mathcal{S}\right\}$, etc.

\section{BIBLIOGRAPHY}

1. P. Billingsly, Ergodic theory and information, Wiley, New York, 1965. MR $33 \# 254$.

2. E. Effros, Transformation groups and $C^{*}$ algebras, Ann. of Math. (2) 81 (1965), 38-55. MR 30 \#5175.

3. D. Freedman, On two equivalence relations between measures, Ann. Math. Statist. 37 (1966), 686-689. MR 33 \#68.

4. P. R. Halmos, Introduction to Hilbert space and the theory of spectral multiplicity, Chelsea, New York, 1951. MR 13,563.

5. - Lectures on ergodic theory, Chelsea, New York, 1960. MR 22 \#2677.

6. K. Kuratowski, Topologie. Vol. I, 3rd ed., PWN, Warsaw, 1952; English transl., PWN, Warsaw; Academic Press, New York, 1966. MR 14,1000; 36 \#840.

7. G. W. Mackey, Borel structure in groups and their duals, Trans. Amer. Math. Soc. 85 (1957), 134-165. MR 19,752.

8. D. Ornstein, Bernoulli shifts with the same entropy are isomorphic, Advances in Math. 4 (1970), 337-352. MR 41 \#1973.

9. - Imbedding Bernoulli shifts in flows, Contributions to Ergodic Theory and Probability (Proc. Conf. Ohio State Univ., Columbus, Ohio, 1970), Springer, Berlin, 1970, pp. 178-218. MR 42 \#7866.

10. D. Ornstein and P. Shields, An uncountable family of $K$-automorphisms, Advances in Math. 10 (1973), 63-88.

11. D. Ornstein and B. Weiss, Finitely determined implies very weakly Bernoulli (to be published).

12. K. R. Parthasarathy, Probability measures on metric spaces, Academic Press, New York, 1967. MR 37 \#2271.

DEPARTMENT OF MATHEMATICS, UNIVERSITY OF CALIFORNIA, BERKELEY, CALIFORNIA 94720 